\title{
Development of Electromagnetic Shielding Wearable Electronic Textiles using Core Conductive Fabrics
}

\author{
C. Vigneswaran \\ Department of Fashion Technology, \\ PSG College of Technology, \\ Coimbatore, India
}

\author{
P. Kandhavadivu \\ Department of Fashion Technology, \\ PSG College of Technology, \\ Coimbatore, India
}

\begin{abstract}
Today wearable electronics are playing a vital role in many applications such as Military, Medical application, telecommunications and health care garments. Conductive textiles also provide wide range of applications in areas of civilian and military purposes. In this research work an attempt has been made to design and develop core-sheath conductive yarns with copper filament and optical fibre as core and cotton as sheath using Dref-3 Friction spinning system. The special guide mechanism has been designed and used to produce uniform structure of core-sheath conductive yarns. Three different core-sheath ratios such as 67/33, $80 / 20$ and 90/10 respectively, were produced by varying the draft in the second drafting unit which fed the cotton carded slivers to cover the core component. Copper filament of 38 SWG British Standard Gauge has been used as core component. The nominal count of core-sheath conductive yarn produced in this research was 328 tex and core component kept as constant 261 tex for all the yarn samples. Optical core conductive yarns are produced by using CK-20 type optical fibres. The process parameters such as perforated drum speed and yarn delivery speed are 4000 revolution per minute and 70 metres per minute respectively, kept as constant for all the samples. The copper core conductive yarns were used to develop the conductive fabrics. These fabrics have very good scope for many applications for development of electromagnetic shielding wearable textiles, mobile phone charging and body temperature sensing garments. It also has the optical fibre core conductive yarns which were used for the development of signal transferring garment for telecommunication and data transferring purpose. In this work two sensors have been integrated in sensorized garments for monitoring the body temperature. Moreover, it has been pointed out the use of these sensorized garments as a valid alternative to existing instrumentations which are in several health care areas. The results on the copper core conductive yarns and Optical core conductive yarns have studied. The characteristics of core conductive fabrics such as air permeability, tensile strength, electromagnetic shielding effect, aerial density, and fabric cover factor have been analysed. The performance of the conductive fabric garment has been briefly reported. The copper core conductive yarn, Optical core conductive yarn, copper conductive fabric and conductive fabric garment have the very high potential which is to be commercialized.
\end{abstract}

Keywords: shielding fabric; core conductive fabric Electronic textiles; copper conductive; Electromagnetic shield

\section{INTRODUCTION}

Outline of friction spinning system, the fibres from the sliver after pre-opening and individualization are deposited in a suitable form by an air current into the gap between two cylindrical drums rotating in the same direction [1]. Either one or both the drums are perforated and have a suction arrangement inside them to restrain the fibres while they are rolled and twisted. The yarn is formed by the frictional forces between the fibres and the rotating cylindrical surfaces and is withdrawn at right angle to the direction of moving surface. Since the drum diameter is many times higher than the yarn diameter, each revolution of drum imparts a large number of turns to the yarn [2]. Dref-3 is a development over Dref-2 for improving the quality of yarn, productivity and core-sheath yarn production. Core-sheath yarn is made to improve the quality of yarn by laying part of the fibres in an aligned fashion along the direction of yarn axis in core. The remaining fibres are wrapped round the core fibres. The sheath fibres are attached to the core fibres by the false twist generated by the rotating action of the drums [3].Two drafting units are, therefore, used in the system, one for the core fibres and other for the sheath material. After drafting in the first unit, the core filament copper fed to nip between the spinning drums in a direction parallel to the axis of the drums. The sheath fibres after passage through the second drafting unit and are deposited over core filaments by the air stream and wrapped over the core by the rotating action of the drums $[4,5]$. The properties of friction -spun yarns, ring-spun yarns and other yarns spun on modern spinning systems have been compared by several authors [7-12] and the findings have been at times divergent because of the differences in raw material and processing conditions. Padmanabhan and Ramakrishnan [8] found that the filament core Dref-3 spun yarn is stronger than $100 \%$ cotton yarn and cotton core yarn by about $13 \%$ and $20 \%$ respectively. Perumalraj et al [14] also reported the production of core-sheath Dref-3 friction spun yarn made out of cotton and polyester sheath and 820 denier copper filaments as core for development of electromagnetic shielding. Many applications in e-textiles have been pointed out by many researchers [15-19] for development of sensor wears, electromagnetic shielding and tele communication using conductive yarns. Ching-wen Lou [24] et al developed a complex core spun yarn using stainless steel as core in the ring spinning system. Also he point out the yarn structure made out of this system not uniform to get perfect core-sheath yarn structure. Wireless communications are impacting all areas of the military, from logistics and training to collaboration and medical support. In military offices, wireless communications keep people mobile, with continual access to information anywhere, any time. Laptop computer users attending meetings and conferences now maintain instant access to data files and internet searching so they can obtain immediate answers to questions raised. Outside the office, mobility increases productivity by allowing users to work in previously unproductive situations, such as while 
traveling or wailing for appointments [20]. An attempt has been taken for development of core-sheath conductive yarn using Dref-3 spinning system, using copper filament (328 tex) as core and MCU cotton fibres (32 $\mathrm{mm}$ staple length) as sheath to produce three different blend ratios of 33/67, 20/80 and 10/90. Further Electro-Mechanical properties of these core-sheath Dref-3 conductive yarns are examined and reported. The Optical core conductive yarns also been produced in the Dref-3 friction spinning system and the garment has been made for signal transferring and telecommunication.

\section{MATERIALS AND METHODOLOGY}

In this chapter, the various materials used for this research work to produce Copper core conductive yarns, Optical core conductive yarns, Copper conductive fabrics and Optical conductive fabrics have been reported. It also dealt with the methodology adopted to produce the products and testing the same.

\subsection{Materials}

The core and sheath components used for manufacturing of conductive yarns are given below:

I. Copper core conductive yarns: In this research work, 38 SWG (British standard wire gauge) copper filament and MCU-5 cotton fibres were used as core material and sheath material respectively.

II. POF core conductive yarns: In this research work, two types of Plastic Optical Fibres (POF) were used as core materials namely ESKA and CK-20 which has diameter of $500 \mu \mathrm{m}$ and $1000 \mu \mathrm{m}$ respectively and MCU-5 cotton fibres as sheath material.

\subsection{Methods}

The Fehrer AG type DREF-3 friction spinning machine was used to produce three different core-sheath ratios of conductive yarns. Four cotton carded slivers were fed in the first drafting unit and each sliver has 4.22 grams per metre and silver irregularity value U\% - 3.8, respectively. The core material (Copper filament- 261 tex) was fed in the first drafting unit with special guides. The guide device was designed and installed on the first drafting unit to increase the stability of spinning of the metal yarn. The process parameters of Dref-3 spinning machine such as perforated drum speed and yarn delivery rate were set at 4000 revolutions per min and $70 \mathrm{~m} / \mathrm{min}$ respectively, to produce uniform yarn structure and which has the nominal yarn count of 328 tex. By varying the draft in the second drafting system, three different coresheath ratios such as $67 / 33,80 / 20$ and $90 / 10$ were produced. The yarn formation in the DREF-3 friction core spun yarn are carried out and for this research work, conductive yarns were produced which has three different core-sheath ratios. During the spinning process, the copper filament was fed from the first drafting unit with special guides. The guide device was designed and installed on the first drafting unit to increase the stability of spinning of the conductive yarn. The special care was taken while wrapping copper filament by adjusting the perforated drum speed, yarn delivery rate and draft in second drafting unit to produce uniform yarn structure.

\subsection{Design and Development of Copper Core Conductive Fabrics}

The copper core conductive fabrics are produced using power loom with copper core conductive yarns as warp and weft threads. The physical characteristics of these core conductive fabrics will be discussed in this paper.

\subsection{Design and Development of Optical Core Conductive Fabrics}

The optical core conductive fabrics are produced using power loom with optical core conductive yarns as weft threads and $2 / 60$ s Ne cotton threads as warp threads. The optical core conductive fabrics of ESKA \& CK-20 type optical fibres respectively. The physical characteristics of these fabrics will be discussed.

\subsection{Material Testing}

The physical characteristics of conductive yarns and fabrics are tested as per standard testing procedures. The testing methodology \& procedures are given below.

\subsubsection{Yarn Testing}

The yarn count, tenacity \& breaking elongation and electrical properties were tested for copper core conductive yarns. The testing procedures of above tests will be discussed in this paper.

\subsubsection{Fabric Testing}

The fabric thickness, aerial density, cover factor, tensile strength, air resistance and electromagnetic shielding effectives were tested for copper core conductive yarns. The testing procedures of above tests will be discussed.

\section{APPLICATIONS OF CONDUCTIVE FABRICS}

In this chapter the various applications of conductive fabrics made out of copper core conductive yarns and optical core conductive yarns have been reported.

\subsection{Copper Core Conductive Fabrics}

The applications of copper core conductive fabrics made out of copper core conductive yarns as warp and weft threads are given below:

* Electromagnetic shielding garment for defence personnel

* Mobile phone charging - wearable electronics development.

* Body temperature sensing garment

$>$ The copper core conductive yarns are used for development of conductive fabrics. In this project two different woven fabric constructions were made to analyze the electromagnetic shielding effectiveness.

$>$ Today smart garments are playing vital role and popular in the e-textiles. These garments are developed with integrated circuits and operating at very low voltages. Many researchers are developing the smart garments with thin film technology and solar power circuits.

$>$ The another application of these conductive fabrics are usable for sensing the body temperature and it can be applicable for medical scanning purpose and heath care areas. 


\subsection{Optical Fibre Core Conductive Fabrics}

The applications of optical core conductive fabrics made out of plastic optical fibres (POF) optical core conductive yarns as weft threads are given below:

* Tele-garment for signal transferring

* Protective garment for defence

* Garment made for body temperature sensing and mobile phone charging (dual purpose) for defence personnel

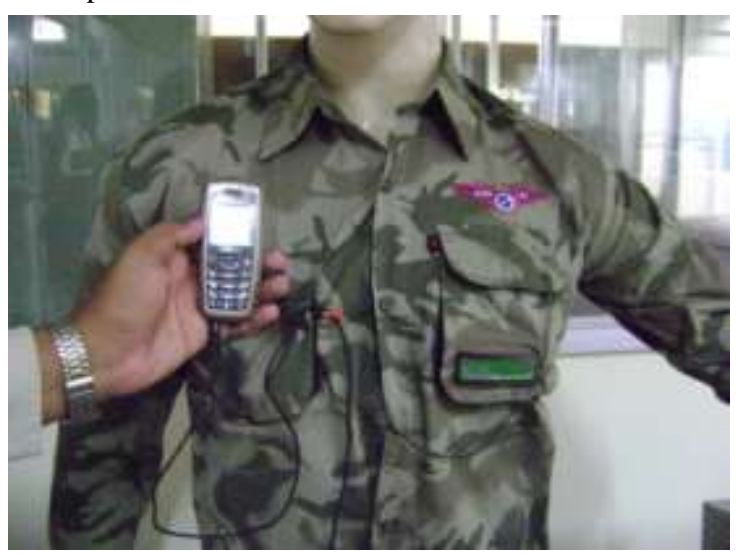

* In this research work, Garment was designed shown in plate 1 with integrated circuit and sensors for body temperature sensing and mobile phone charging (dual purpose) for defence personnel. The garment has designed and embedded with two internal temperature sensors with $\mathrm{I} / \mathrm{C}$.

\section{RESULTS AND DISCUSSION}

In this chapter the various test results obtained by copper core conductive and optical core conductive yarns have been reported such as Contribution ratio of core-sheath components, Tenacity and breaking elongation and electrical properties. Also the electro-mechanical characteristics of conductive fabrics made out of copper core yarn and optical core yarns have been reported

\subsection{Contribution Ratio of Core-Sheath Components}

The core and sheath contribution and core-sheath interaction factors to tenacity are derived from the following equations [13]:

Core contribution factor $\left(\mathrm{CC}_{\mathrm{T}}\right), \%=(\mathrm{X} / \mathrm{Z}) \times 100$

Sheath Contribution factor $\left(\mathrm{SC}_{\mathrm{T}}\right), \%=(\mathrm{Y} / \mathrm{Z}) \times 100$

Core-sheath interaction factor $\left(\mathrm{CSI}_{\mathrm{T}}\right), \%=[(\mathrm{Z}-\mathrm{X}-\mathrm{Y}) / \mathrm{Z}] \times 100$

Where $\mathrm{X}, \mathrm{Y}$ and $\mathrm{Z}$ represent the tenacity of the individual core component and parent Dref-3 yarn, respectively. The fibre strength exploitation is represented as the ratio of tenacity of yarn, core, or sheath component to fibre tenacity, expressed as a percentage.
Table 1. Structural parameters of parent dref-3 friction spun yarn and core-sheath components.

\begin{tabular}{|c|c|c|c|c|c|}
\hline \multirow[t]{2}{*}{$\begin{array}{c}\text { Sample } \\
\text { code }\end{array}$} & \multirow{2}{*}{$\begin{array}{c}\text { Core } \\
\text { Copper } \\
\text { (gpm) }\end{array}$} & \multirow{2}{*}{$\begin{array}{c}\text { Sheath } \\
\text { Cotton } \\
(\mathrm{gpm})\end{array}$} & \multirow{2}{*}{$\begin{array}{c}\text { Parent } \\
\text { yarn } \\
(\mathrm{gpm})\end{array}$} & \multicolumn{2}{|c|}{$\begin{array}{c}\text { contribution } \\
\%\end{array}$} \\
\hline & & & & Core & Sheath \\
\hline Sample A & 0.261 & 0.13 & 0.391 & 66.75 & 33.25 \\
\hline Sample B & 0.261 & 0.058 & 0.319 & 81.82 & 18.18 \\
\hline Sample C & 0.261 & 0.026 & 0.287 & 90.94 & 9.06 \\
\hline
\end{tabular}

Note: gpm- grams per metre

Table 2. Parent dref-3 linear density and diameter of coresheath components.

\begin{tabular}{|c|c|c|c|c|}
\hline \multirow{2}{*}{$\begin{array}{c}\text { Sample } \\
\text { code }\end{array}$} & \multicolumn{2}{|c|}{ Diameter, mm } & \multicolumn{2}{c|}{ linear density, tex } \\
\cline { 2 - 5 } & $\begin{array}{l}\text { Copper } \\
\text { filament }\end{array}$ & $\begin{array}{l}\text { Parent } \\
\text { yarn }\end{array}$ & $\begin{array}{l}\text { Parent } \\
\text { yarn }\end{array}$ & Copper \\
& filament \\
\hline Sample A & 0.1524 & 0.738 & 391.05 & 261 \\
\hline Sample B & 0.1524 & 0.667 & 319.36 & 261 \\
\hline Sample C & 0.1524 & 0.632 & 286.65 & 261 \\
\hline
\end{tabular}

\subsection{Tenacity and Breaking Elongation}

The tensile properties of the parent conductive Dref-3 yarn and their core and sheath components are determined using USTER TENSORAPID Model 3 v7.0. The yarn gauge length and traverse speed of the instruments was set at $500 \mathrm{~mm}$ and $5000 \mathrm{~mm}$ per min respectively. The breaking tenacity and elongation $\%$ of conductive yarns are tested. It is clear that the major strength of yarn is being shared by core portion and sheath portion, due to their different structural parameters of the sheath component. It is also evident from Table 2 that with increased blend ratio of sheath component, contribution of the core component in tenacity values shows increasing trend.

Table 2. shows that the breaking elongation of the individual sheath component for all the samples are almost of a magnitude similar to that of the parent Dref-3 yarn, whereas in the case of the core component it breaks immediately. It is also observed that the sample A (67/33 core-sheath ratio) shown improved elongation to break when compare to other core-sheath yarns due to improvement in core-sheath interaction $\left(\mathrm{CSI}_{\mathrm{T}}-21.22 \%\right)$.

\subsection{Electrical Properties}

Electrical properties of core-sheath conductive Dref-3 spun yarns such as electrical resistance and current in amps are measured at different applied voltage of $6 \mathrm{v}, 12 \mathrm{v}$ and $24 \mathrm{v}$. The values are given in Table 3 . It shows that the electrical resistance of conductive yarns is varying according to the 
length of the yarn and current in amps also directly proportional to the applied voltage. The current values of conductive yarns are decreasing in trend as the yarn length increases; it is due to increasing electrical resistance of the conductive yarns.

Table 3. Electrical properties of core-sheath conductive dref-3 spun yarn

\begin{tabular}{|c|c|c|c|c|}
\hline \multirow{2}{*}{$\begin{array}{c}\text { Conductive } \\
\text { Yarn } \\
\text { length, } \mathbf{m}\end{array}$} & \multirow{2}{*}{$\begin{array}{c}\text { Electrical } \\
\text { Resistance, } \\
\mathbf{M} \boldsymbol{\Omega}\end{array}$} & $\begin{array}{c}\text { Applied } \\
\text { voltage } \\
\mathbf{6 V}\end{array}$ & $\begin{array}{c}\text { Applied } \\
\text { voltage } \\
\mathbf{1 2 V}\end{array}$ & $\begin{array}{c}\text { Applied } \\
\text { voltage } \\
\mathbf{2 4 V}\end{array}$ \\
\cline { 3 - 5 } & $\begin{array}{c}\mathbf{C u r r e n t} \\
(\boldsymbol{\mu} \mathbf{A})\end{array}$ & $\begin{array}{c}\text { Current } \\
(\boldsymbol{\mu} \mathbf{A})\end{array}$ & $\begin{array}{c}\text { Current } \\
(\boldsymbol{\mu} \mathbf{A})\end{array}$ \\
\hline 0.5 & 0.06 & 100 & 200 & 400 \\
\hline 1 & 0.1 & 60 & 120 & 240 \\
\hline 2 & 3 & 2 & 4 & 8 \\
\hline 5 & 20 & 0.3 & 0.6 & 1.2 \\
\hline 10 & 28 & 0.21 & 0.42 & 0.84 \\
\hline
\end{tabular}

\subsection{Physical Characteristics of Conductive Fabrics Made Out Of Copper Core Yarn and Optical Core Yarns}

The Physical characteristics of conductive fabrics such as aerial density, fabric cover factor, ends per inch, picks per inch, fabric strength, fabric thickness and air resistance values are made as per standard test procedures. The two different structures of copper and optical core conductive fabrics are produced and the particulars of fabric thickness and air permeability have also reported.

\subsection{Electromagnetic Shielding Tests:}

Electromagnetic shielding is the process of limiting the coupling of an electromagnetic field between two locations. The shielding can be achieved using a material acting as a barrier. The EMSE of the conductive fabric was measured as per ASTM D4935-99 Test method for measuring the electromagnetic shielding effectiveness of planar materials with the given set up.

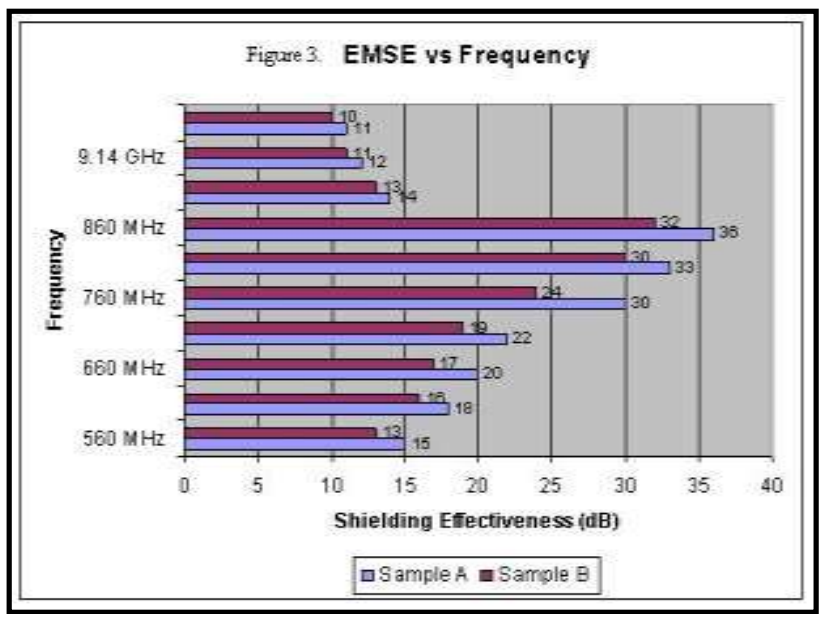

Figure 1. EMSE vs frequency of conductive fabrics.

The electromagnetic shielding effectiveness of the copper conductive fabrics are made and the effectiveness was measured between the ranges of $560 \mathrm{MHz}$ and $9.22 \mathrm{GHz}$. The figure 1 shows an attenuation of about $32 \mathrm{~dB}$ on an average for all frequencies from $760-860 \mathrm{MHz}$ for both sample A (CCYwarp and weft) and sample B (CCY-weft only). It can also be inferred from the graph that the sample A with core-sheath ratio of 67/33 exhibits a highest shielding effect in the range $760-860 \mathrm{MHz}$. It is suggested that the sample A and B can be used to shield television, computers and similar equipments and also shielding gadgets like cellular phones. The variations in the shielding effectiveness of the fabrics can be contributed to the fact that the electrical property of the material varies depending upon the frequency. So it is suggested to use the fabric at frequencies where higher attenuation is obtained. The shielding effectiveness can be increased further by increasing the cover factor of the fabric.

\section{CONCLUSION}

Through this research work the following conclusions have been made:

- The copper core conductive yarns were produced using Dref-3 friction Spinning System with Copper filament of 261 tex as core and MCU-5 cotton fibres as sheath. For the above core-sheath yarn manufacturing, a special guide mechanism has been developed to maintain the copper filament tension and to produce uniform yarn structure.

- Three different core-sheath ratios of $67 / 33,80 / 20$ and 90/10 were used to produce copper core conductive yarns by varying the draft in the second drafting unit. Among the three core-sheath ratios, the $67 / 33$ core-sheath conductive yarn has the highest tenacity of $3.27 \mathrm{cN} /$ tex and elongation to break $5.27 \%$ when compare to other core-sheath ratios. The technical reason for the same is because of its better core-sheath interaction factor $\mathrm{CSI}_{\mathrm{T}}$ $21.22 \%$. It is also observed that the core-sheath interaction factor of conductive yarn 90/10 core-sheath component was found low core-sheath interaction factor $\mathrm{CSI}_{\mathrm{T}}-9.21 \%$ which causes the lower breaking tenacity and elongation percent.

- The optical core conductive yarns were produced using Dref-3 Spinning System with ESKA \& CK-20 type plastic optical fibres (POF) as core and MCU-5 cotton fibres as sheath.

- The interaction of core-sheath behavior and mechanical properties of the Dref-3 conductive yarn are mainly depends upon the frictional characteristics and the percentage of core-sheath components. The electrical properties of these conductive core yarns have been studied at three differed applied voltages of $6 \mathrm{v}, 12 \mathrm{v}$ and $24 \mathrm{v}$. It is found that the core-sheath yarns have very low resistance ranges 3-28 $\mathrm{M} \Omega$.

- The copper core conductive fabrics and optical core conductive fabrics were produced using the copper core conductive yarns and optical core conductive yarns.

- The electro-mechanical characteristics of these conductive fabrics are studied.

- A sensorized and signal transferring garment has been designed and developed using the copper and optical core conductive fabrics for monitoring the body temperature, mobile phone charging and signals transferring. The performances of sensorized and signal transferring garment have also studied. 


\section{ACKNOWLEDGEMENT}

We extend our sincere thanks to The Management of PSG Institutions for providing the necessary infrastructural facilities for carrying out our project work successfully. We wish to express our sincere thanks to Dr.R.Rudramoorthy, Principal, PSG College of Technology for providing us with the necessary facilities in carrying out our research work, continuous support and encouragement.

\section{REFERENCES}

[1] Balasubramanian, N. 1992. Friction Spinning - A critical review, Indian J Fibre Text Res, 17, 246-251.

[2] Gruoner, S., 1977. The Dref spinning system, Text Asia, 8(3), 44-56.

[3] Lord, P.R., Joo, C.W., and Ashizaki, T. 1987. The Mechanics of friction spinning, J.Text. Inst. 78 (4), 23439.

[4] Rust, J,P., and Lord, P.R., 1991. Variations in yarn properties caused by a series of design changes in friction spinning machine. Text. Res. J. 61, 645-656.

[5] Krause, H,W., Solomon, H,A., and Stalder, H. 1989. The yarn formation in friction spinning. Int. Text. Bull. Yarn forming. 4, 31-37.

[6] Lord, P.R., and Rust, J.P., 1991. Fibre assembly in friction spinning. J.Text. Inst. 82, 465-471.

[7] Louis, G.L, Salaun, H.L., and Kimmel, L.B., 1985. Comparison of properties of cotton yarns produced by Dref 3, Ring and Open end spinning methods, Text. Res. J. 55,344 .
[8] Padmanabhan, A.R., and Ramakrishnan, N. 1991. Properties of Man-made fibre yarns spun on Dref 3 spinning system. Ind. J. Fibre. Text. Res. 16, 241-248.

[9] Gsteu, M., 1989. High tech yarns with friction spinning technology, Int. Text. Bull, Yarn forming, 1, 36-48.

[10] Chattopadhyay, R., Salhotra, K.R., and Dhamija, S. 2000. Influence of core-sheath ratio and core type on Dref-III friction spun core yarns, Ind. J Fibre. Text. Res. 25, 256-263.

[11] Ishtiaque, S.M., and Agarwal, D., The Internal structure of sheath fibre in Dref-3 yarn. J. Text. Inst. 91, 546-562.

[12] Tyagi, G.K., Kaushik, R.C.D., Dhamija, S., Biswas, B., and Salhotra, K.R. 1999. Influence of core content and friction ratio on properties of Dref-3 polyester yarns, Ind. J. Fibre. Text. Res., 24, 183-187.

[13] Das, A., Ishtiaque, S.M., and Yadav, P. 2004. Contribution of core and sheath components to the tensile properties of Dref III yarn, Text. Res. J, 74 (2), 134-139.

[14] Perumalraj, R.,, Dasaradan, B.S., and Sampath, V.R. 2007. Study on copper core yarn conductive fabrics for Electromagnetic shielding, National conference on Functional Textiles and Apparels, 2, 187-203.

[15] Ashokkumar, L., and Venkatachalam, A. 2007. Electrotextiles: concepts and Challenges, National conference on Functional Textiles and Apparels, 2, 7584.

[16] www.electrotextiles.com 